\title{
Behandlung von suchtkranken Menschen mit komplexen Traumatisierungen in der Fachklinik Richelsdorf
} H. Hinz

\author{
Treatment of Addicts with Complex Trauma at the Specialised \\ Hospital Richelsdorf
}

\section{Einleitung}

Die größte Gruppe der PatientInnen mit schweren Traumatisierungen in der Fachklinik Richelsdorf sind Frauen. Etwa ein Drittel unserer Patientinnen sind in Kindheit oder Jugend Opfer sexueller Übergriffe von Angehörigen (Väter, Stiefväter, Brüder o.Ä.) gewesen. In der letzten Zeit steigt die Zahl der männlichen Patienten, bei denen wir eine PTBS nach Missbrauchs- oder Gewalterfahrungen diagnostizieren.

Menschen, die schwere oder längerfristige Traumatisierungen erfahren haben, leben mit einem überdauernden Gefühl von Hilflosigkeit. Die Lebensgeschichte bedingt eine Erwartungshaltung, die zu einer Wiederholung der Traumata im weiteren Leben führt. Verdrängung wäre der Abwehrmechanismus, der am wenigsten zu krankhaften seelischen Zuständen führt. Meist gelingt der Verdrängungsvorgang nicht und die Seele muss zu weniger erfolgreichen Abwehrmechanismen greifen (zu Spaltung, Depersonalisation- oder Derealisation) oder zur Einnahme von Stoffen, die die Wahrnehmung verändern und betäuben - Drogen, Alkohol, Medikamente, die letztendlich in die Sucht führen. Eine andere Möglichkeit, das Ich notdürftig stabil zu halten, ist selbstverletzendes Verhalten.

Wie behandeln wir suchtkranke PatientInnen mit Missbrauchserfahrungen?

Zentraler Inhalt der Therapie ist die psychische Stabilisierung. Das therapeutische Klima ist gekennzeichnet von Respekt und der sorgfältigen Vermeidung von Grenzüberschreitung. Informa- tion und Aufklärung der PatientInnen über die Erkrankung und das therapeutische Vorgehen unterstützen die Selbstsicherheit.

Da ein Aufdecken der Missbrauchserfahrung in der Regel eine Retraumatisierung bedeutet und daher nicht hilfreich ist, bevor eine sehr tragfähige, stabile therapeutische Beziehung entstanden ist und die aktuelle Lebenssituation missbrauchsfrei ist, wird auf Konfrontation mit dem Trauma und konfrontative Techniken bewusst verzichtet. Das Gespräch über die traumatischen Erfahrungen findet ausschließlich in der therapeutischen Einzelsitzung statt, um Triggerreize zu vermindern und eine wohldosierte Auseinandersetzung und Kontrolle der emotionalen Lage sicherzustellen.

Somatisierungsstörungen gehören zu dem Erkrankungsbild und werden den PatientInnen erklärt, um Akzeptanz und Verständnis für die eigene Hypersensibilität zu wecken und dadurch psychisch zu entlasten und zu stärken. Linderung der körperlichen Beschwerden wird mit Verfahren aus der Naturheilkunde, Entspannungsverfahren und physiotherapeutischen Maßnahmen erreicht. Körperorientierte Entspannungsverfahren (Muskelentspannung nach Jakobsen, Tai Chi), regelmäßige Sport- und Bewegungstherapie und Training an den Fitnessgeräten zur Verbesserung der Körperwahrnehmung und der Körpergrenzen (gerade auch bei Menschen, die sich selbst verletzen müssen) unterstützen dabei ebenso wie imaginative Übungen.

Da die aktuelle Beziehungsgestaltung durch die Missbrauchserfahrung geprägt ist, ist es uns wichtig, im therapeutischen Setting mit den PatientInnen vorsichtig, maßvoll und ihre Schwierigkeiten verstehend, Abgrenzung und Grenzsetzung einzuüben und damit das Ich und die Beziehungssicherheit zu stärken. Ge- 
meinsam suchen wir Situationen, die relativ harmlos, unbedeutend und in der Klinik häufig sind und spielen - zunächst gedanklich - Reaktionsmöglichkeiten durch, die aus der Opferrolle heraustreten lassen. Im Klinikalltag probieren die PatientInnen, auch mithilfe der MitpatientInnen ihrer Therapiegruppe aus, welche Fortschritte möglich sind und welche Ängste entstehen. Aktives abgrenzendes Verhalten lässt sich dann weiter übertragen, wenn es gut geht, auch in den privaten Beziehungsbereich bei Besuchen von Angehörigen oder Heimfahrten.

Eine unserer Patientinnen, die von ihrem Vater und dann von ihrem Stiefvater missbraucht wurde, heiratete einen Mann, der sich als gewalttätig herausstellte, trennte sich von ihm und nahm dann nur noch Beziehungen zu Frauen auf. Erschreckenderweise musste sie dabei erleben, dass sich die gleichen gewalttätigen Strukturen in ihrer lesbischen Beziehung wiederholten. Sie floh schließlich mit ihren Kindern ins Frauenhaus. Auch in der aktuellen Beziehung wurde sie von ihrer Partnerin gequält. Sie fühlte sich dem hilflos ausgeliefert, hatte keine Möglichkeiten, sich zu wehren, außer zu fliehen und damit auf die lebensnotwendige Erfahrung von Nähe zu einem anderen Menschen zu verzichten. In der Klinik zog sie sich zunächst in der Freizeit in unsere Obstplantage zurück. Im Laufe der Zeit lernte sie immer besser, sich durchzusetzen, konnte sich in einen Liegestuhl zu den anderen in den Garten legen, ohne sich bedroht zu fühlen, lernte, sich zu verteidigen. Diese Fähigkeiten konnte sie schließlich, bei deren häufigen Besuchen in der Klinik, auch im Kontakt mit ihrer Freundin einsetzen. Es stellte sich zu ihrem Erstaunen heraus, dass die Freundin eine eigentlich eher schüchterne, ängstlich-unsichere Person war, die ihr kaum etwas entgegenzusetzen hatte und der sie keineswegs hilflos ausgeliefert war.

Jahre nach der Entlassung geht es der Patientin jetzt gut und sie ist stabil abstinent. Sie hat Beziehungen, ertappt sich aber immer mal wieder bei zu geringer Selbstfürsorge und Aufgabe ihrer eigenen Wünsche zu Gunsten eines anderen Menschen. Sie berichtet davon, wie sie über sich den Kopf schüttelt und dann korrigiert: „So nicht mehr mit mir“.

\section{Über die Fachklinik Richelsdorf}

Die Fachklinik Richelsdorf wurde vor mehr als 20 Jahren zunächst als kleine Einrichtung für Kurzzeitentwöhnungsbehandlungen eröffnet. Jetzt hat sie 75 Betten und die Behandlungszeiten sind flexibel zwischen 8 und 16 Wochen gestaltbar, werden individuell mit der Patientin und dem Patienten festgelegt. Um den Kontakt zum Therapeuten möglichst vertrauensvoll und sicher zu gestalten, werden die PatientInnen vom ersten Tag ihres Aufenthaltes in der Klinik in ihre Gruppe aufgenommen. Auf eine Aufnahmephase in einer Aufnahmestation wird verzichtet. Die bestehende und funktionierende Gruppe bietet Schutz und Halt. Eine Mitpatientin oder ein Mitpatient aus der Therapiegruppe übernimmt als sog. „Patin“ oder „Pate“ die Einführung und ist in der Anfangszeit insbesondere Ansprechpartner. Der
Gruppentherapeut ist gleichzeitig auch Einzeltherapeut, um die Vertrauensbasis zu stärken.

Es werden alkohol-, medikamentenabhängige und polytoxikomane Frauen und Männer behandelt. Das Therapiekonzept ist in seiner Basis tiefenpsychologisch fundiert, systemische und verhaltenstherapeutische Anteile werden integriert. Um regressive Effekte zu vermeiden, besteht eine deutliche Trennung zwischen Therapie- und Realraum [1]. Im Therapieraum (Gruppen- und Einzelpsychotherapie) wird interpretiert und analysiert, ansonsten werden in allen anderen therapeutischen Bereichen reale Beziehungen hergestellt, an denen Beziehungsaufnahme und angemessener Kontakt geübt werden können.

Seit Mitte der 90er-Jahre wurde unser Augenmerk auf posttraumatische Belastungsstörungen bei Frauen mit Missbrauchserfahrungen gelenkt [2] und ein Konzept entwickelt, das die Erkenntnisse der modernen Traumaforschung berücksichtigt [3-8]. PatientInnen mit posttraumatischen Belastungsstörungen werden nicht in einer gesonderten Gruppe behandelt, um die Einhaltung des Gebots, im alltäglichen Kontakt und in der Gruppe nicht über das Trauma zu sprechen, zu erleichtern und eine Identifizierung über die Traumaerfahrung zu verhindern. Jeder einzelne Psychotherapeut ist unterwiesen und fortgebildet im Umgang mit dieser Erkrankung. Die Therapeuten der Begleittherapie, die Ärzte und das Pflegepersonal kennen sich mit der Erkrankung aus und achten darauf, Grenzüberschreitungen, aber auch (gut gemeinte) therapeutische Übergriffe zu vermeiden, berücksichtigen die Soziophobie, schützen und entlasten die PatientInnen, so dass der gesamte therapeutische Rahmen stabilisierend und stützend wirkt.

\section{Literatur}

${ }^{1}$ Hinz H. Analytisch orientierte Kurzzeittherapie. Schriftenreihe des Fachverband Sucht e.V. 1998; 21: $303-308$

${ }^{2}$ Hinz H. Frühe Missbrauchserfahrungen bei alkoholabhängigen Frauen

- Bedeutung für die aktuelle Lebens- und Beziehungsgestaltung Behandlungsstrategien. Schriftenreihe des Fachverband Sucht e.V. 2004; 27: 96 - 104

${ }^{3}$ Reddemann L, Sachsse U. Imaginative Psychotherapieverfahren zur Behandlung in der Kindheit traumatisierter Patientinnen und Patienten. Psychotherapeut 1996; 41: 169-174

${ }^{4}$ Sachsse U. Die Psychodynamik der Borderlinepersönlichkeitsstörung als Traumafolge. Forum Psychoana 1990; 6: 199-212

${ }^{5}$ Sachsse U. Patienten. Opfer oder Täter? In: Bell K, Höhfeld K (Hrsg). Aggression und seelische Krankheit. Gießen: Psychosozialverlag, 1996: $259-273$

${ }^{6}$ Sachsse U. Selbstverletzendes Verhalten: Psychodynamik - Psychotherapie; das Trauma, die Dissoziation und ihre Behandlung. Göttingen, 1996

7 Sachsse U, Eßlinger K, Schilling L. Vom Kindheitstrauma zur schweren Persönlichkeitsstörung. Fundamenta Psychiatrica, 1997: 20: 19-27

8 Tameling A, Sachsse U. Symptomkomplex, Traumaprävalenz und Körperbild von psychisch Kranken mit selbstverletzendem Verhalten. Zeitschrift für Psychosomatik, Psychotherapie, medizinische Psychologie 1996; 46: $61-67$ 